\section{Fronteras como categoría social. La perspectiva teórica de las fronteras en el siglo XXI}

\section{Borders as a social category. A theoretic perspective on borders in the $21^{\text {st }}$ century}

\author{
Karol Derwich* \\ DEPARTAMENTO DE ESTUDIOS LATINOAMERICANOS \\ UNIVERSIDAD JAGUELÓNICA, CRACOVIA \\ $\triangle$ karol.derwich@uj.edu.pl \\ https://orcid.org/0000-0001-8438-8601
}

Anuario Latinoamericano Ciencias Políticas

y Relaciones Internacionales

vol. 6, 2018

pp. $37-51$

DOI: $10.17951 /$ al.2018.6.37-51

\title{
RESUMEN
}

Junto con la intensificación significante del proceso de globalización, entramos al mundo en el cual las fronteras nos afectan en muchas situaciones. La globalización no conduce al mundo sin fronteras. Más bien, las fronteras están más cerca de los individuos que pueden tener más facilidad para cruzarlas. Este artículo ofrece una breve revisión de las teorías contemporáneas de fronteras, pero también es una prueba de explicar el sentido de percibir fronteras principalmente como fenómeno social. Según el autor, este enfoque permite comprender mejor el funcionamiento de las fronteras en el mundo contemporáneo.

PALABRAS CLAVE: fronteras, globalización, zonas fronterizas, muros.

\begin{abstract}
With a significant intensification of the process of globalization, borders seem to affect individuals in many situations. Globalization does not lead to a borderless world. On the contrary, borders are nearer to individuals that can cross them in an

* Profesor asociado en el Departamento de Estudios Latinoamericanos de la Universidad Jaguelónica en Cracovia, Polonia. Doctor en Ciencias Políticas. Su área principal del trabajo académico es el tema del Estado: disfunción del Estado, caída del Estado, regímenes políticos y sus transformaciones. Autor de varios libros y artículos. Sus últimas publicaciones: Meksyk - między demokracją a dysfunkcyjnością, Universitas, Kraków 2017; Sistema político como la fuente de disfunción del Estado en México, en: Derechos Humanos y Seguridad en Democracia, A. Villareal Palos, J. Chaires Zaragoza, D. J. Haro Reyes (eds.), Universidad de Guadalajara, Guadalajara 2017; La transformación interminable. El caso difícil de la democratización en México, en: Transiciones políticas contemporáneas. Singularidades nacionales y dinámica general del cambio, C. González Martínez (ed.), Fondo de Cultura Económica, Universidad de Murcia 2018.
\end{abstract}


Dossier América Latina: fronteras y zonas fronterizas easier way. The article is a brief revision of contemporary theories of borders, but the author also tries to explain the sense of perceiving borders mainly as a social category. According to the author, this approach allows a better understanding of borders' functioning in the present world.

KEYWORDS: borders, globalization, border zones, walls.

\section{Introducción - la globalización y el Estado nación}

A finales del siglo XX el proceso de globalización se intensificó enormemente. A pesar de que este proceso se inició mucho más antes, especialmente el cambio del siglo XX al XXI fue un tiempo en el que los procesos de globalización tomaron gran velocidad y afectaron muchas esferas de la vida tanto de los hombres como de los sujetos de las relaciones internacionales. Esta intensificación del proceso de globalización tuvo gran impacto sobre el funcionamiento de los actores principales del sistema internacional, es decir, los Estados. Sin duda, los cambios resultantes de la globalización tienen gran importancia y sus efectos se pueden observar en muchas áreas del funcionamiento tanto del sistema internacional como en la misma institución del Estado. Se puede decir que el proceso de globalización está presente en el mundo desde la era de los descubrimientos, cuando se construyeron las conexiones entre varias partes del mundo que antes estaban separadas. Pero no se debe buscar en los siglos tan lejanos. Si se comparara el mundo del siglo dieciocho con el de ahora, se podrían ver fácilmente las diferencias. En el sentido económico, el mundo contemporáneo está mucho más integrado. Gracias a los bajos costos del comercio, los consumidores pueden comprar los bienes que quieren de manera mundial. Así pueden vender sus productos los productores. Aunque, cuando se observa el mundo contemporáneo, se puede reflexionar que en el sentido político todavía está muy dividido y desintegrado (Ben G. Li, Penglong Zhang 2017). El Estado-nación todavía es el sujeto principal del sistema internacional. En este sentido se puede comentar que el sistema construido sobre la base de la Paz de Westfalia todavía funciona. Pero con un análisis más detallado se puede observar el constante cambio y evolución del sistema de Westfalia. Unos académicos decidieron anunciar la aparición -en consecuencia de la globalización- del sistema postwesfaliano. El argumento que apoya esta postura es el gran crecimiento del papel de los actores no estatales y su influencia sobre las relaciones internacionales. Se debe estar de acuerdo con la opinión de que los actores no estatales juegan un papel mucho más importante en el sistema internacional y que su impacto en los Estados es mucho más importante. No obstante, los últimos años nos muestran que en las situaciones de crisis -sean económicas, políticas o, como últimamente se puede observar en los países europeos, migratorias- todos ponen su atención al Estado. Todavía ciertas necesidades de los ciudadanos pueden ser atendidas solamente por las 
instituciones estatales. En consecuencia, parece que la evolución del sistema westfaliano nos lleva al modelo -usando el lenguaje del siglo XXI- del sistema wesfaliano 2.0, o sea, modificado, pero todavía basado en el papel principal de los Estados. Sin duda, en comparación con los siglos anteriores, a la vuelta de los siglos XX y XXI cambió la situación del Estado en el sistema internacional. Como consecuencia de transformaciones tan importantes, el papel y las tareas del Estado cambian. Pero no en una manera suficiente como para formular una tesis sobre el fin del Estado (Held, McGrew, Goldblatt, Perraton 1999: 489; Zachara 2012: 95). Como el actor de las relaciones internacionales, el Estado está evolucionando y buscando nuevas posibilidades de funcionamiento en el modelo del sistema internacional modificado. Todavía es el actor más importante, aunque los retos por parte de actores no estatales crecen constante y considerablemente. Vale la pena señalar que las guerras más conocidas del siglo presente son la guerra contra el terrorismo y la guerra contra las drogas. La amenaza mencionada más frecuente en las estrategias de seguridad no son otros Estados sino los grupos terroristas. Las corporaciones trasnacionales a veces tienen posibilidades de influir en las políticas de Estados particulares de una manera muy efectiva. Obviamente, las posibilidades de funcionamiento de los actores no estatales y simultáneamente transnacionales se ampliaron en el efecto de la globalización. Estamos de acuerdo con Moisés Naím, quien escribió que la intensificación del comercio internacional y los vínculos más cercanos entre los Estados fueron percibidos como un remedio y una medida preventiva contra los conflictos internacionales, pero al mismo tiempo, las facilidades en comunicaciones, el transporte, el movimiento de bienes, las personas y servicios construyeron condiciones perfectas para los sujetos no estatales. Estos sujetos, muchas veces competitivos a los Estados, están usando los beneficios de globalización de manera perfecta. En efecto, algunos de estos sujetos llegan a ser desafíos importantes para los Estados. El poder está fluctuando de los gobiernos a las organizaciones no gubernamentales. Las esperanzas de que la distancia y la geografía pierdan su valor no se cumplen (Naím 2009: 30). En este contexto también apareció el término "mundo sin fronteras" (borderless world). Se enfoca en la disminución no solo del concepto del Estado-nación, sino también en el rechazo del territorio como una base del poder o identidad. La ubicación geográfica pierde su importancia. Se comenta que los Estados son cada vez más capaces de monitorizar y controlar lo que entra a su territorio (Rosenau 1997: 4-5). Otra vez se puede referir a la situación del mundo en los últimos años de la segunda década del siglo presente y señalar que cuando se identifican peligros para los ciudadanos del Estado particular, el tema de sus fronteras siempre vuelve. Así es en el caso del peligro del terrorismo en algunos países de Europa occidental, que por razones de seguridad restringieron la posibilidad de cruzar sus fronteras. Cuando apareció la ola de migraciones en Europa en el año 2015, varios gobiernos decidieron cerrar sus fronteras o, al menos, limitar significativamente las posibilidades de entrar a su territorio. En la campaña electoral en los Estados Unidos en 2016,
Fronteras como categoría social. La perspectiva teórica de las fronteras en el siglo XXI

Karol Derwich 
Dossier América Latina: fronteras y zonas fronterizas el candidato a la presidencia estadounidense Donald Trump mostró el muro en la frontera con México como un remedio a los problemas internos de su país, tales como seguridad, desempleo o competencia de fuerza laboral de los inmigrantes mexicanos. En este sentido, la práctica de las relaciones internacionales contemporáneas nos muestra, de manera muy obvia, que el mundo sin fronteras no existe. La globalización no es el proceso que tira las fronteras al basurero de la historia. Las fronteras, como límite del poder de una autoridad, están vinculadas inseparablemente al territorio del Estado. Y así como no hay Estados sin población, de la misma manera no hay Estados sin territorio, el cual tiene su límite. Porque la globalización no es el proceso en contra de los Estados. De hecho, como explica Joseph Stiglitz, es el proceso de integración de los Estados y la gente, el cual es un efecto de la gran reducción de los costos de transporte y telecomunicación, y también la eliminación de las barreras artificiales en la circulación de bienes, servicios y personas. Este proceso está acompañado por la creación de instituciones internacionales, cuyas actividades tienen carácter transnacional (Stiglitz 2004: 26). Sin embargo, se debe recordar que tanto en el caso de las nuevas reglas del comercio como en el de las instituciones internacionales, tales como el Fondo Monetario Internacional, los sujetos principales son los Estados. El mundo está dividido entre unidades nacionales en efecto de las políticas y la creciente integración. Los vínculos económicos no van a cambiar esta situación. Los gobiernos pueden tolerar un nivel más o menos alto de pérdida de su influencia económica en nombre del crecimiento y del desarrollo de su país, pero no van a aceptar ninguna forma de una limitación de su existencia (Kissinger 2002: 217). Tomando esto en cuenta, vale la pena analizar el tema de las fronteras en el mundo globalizado.

\section{Fronteras en el mundo contemporáneo}

Sin duda, las fronteras cambian su naturaleza y su carácter. Las fronteras de los reinos europeos del siglo XVI fueron muy diferentes de las fronteras de hoy día. La diferencia no es solo en la geografía y la forma de estas fronteras. También el entendimiento y la percepción de fronteras en siglo XXI es bastante diferente. Pero todavía no se puede imaginar el mundo sin fronteras, a pesar de su naturaleza diferente. Todavía las fronteras y el concepto de territorio constituyen la base para diferenciar a las comunidades. Son uno de los factores que construyen la identidad colectiva. La posibilidad de contrastar una comunidad con las de afuera, "nosotros" versus "otros", es la base de la existencia de las sociedades (Walther, Retaillé 2014: 3). En el siglo XIX, las fronteras eran percibidas como líneas físicas y visibles que separaban los Estados. Fueron creadas para efecto de actividades diplomáticas de los Estadosnación como ejemplificación o confirmación de su soberanía. Mientras que las fronteras modernas no son solamente las líneas que separan unos Estados de otros. No funcionan de la misma manera que antes, al menos no en todos 
los aspectos de su funcionamiento. La globalización de la cultura, la internacionalización de la economía y de la política, la caída de la Unión Soviética y el bloque de los Estados socialistas resultaron en un proceso de apertura de las fronteras (Donnan, Wilson 2001: 18). Aunque se debe recordar que este proceso tiene varios niveles en varias regiones y situaciones. Todavía se pueden observar fronteras cerradas totalmente. Existen situaciones en las que el manejo de una frontera depende de la política particular y de las tendencias existentes entre las sociedades y los que tienen el poder. Hoy día es mucho más propio percibir las fronteras como un proceso. Por eso a veces se puede encontrar con la noción de "proceso de fronterización" ("process of bordering") (Newman 2006: 148). Las tecnologías nuevas han cambiado las fronteras. Las guerras modernas, los medios de comunicación, el transporte, etc., ahora son más independientes de las fronteras. Tanto los bienes y servicios como las personas pueden cruzar fronteras con mucha facilidad. El sistema bancario internacional, los movimientos internacionales de migrantes laborales y el comercio influyen en la economía tanto a nivel mundial como a nivel de países particulares. Como resultado de estos cambios, las fronteras se vuelven mucho más permeables (Newman 2006: 144; Anderson, O’Dowd 1999: 597-598).

Tomando esto en consideración, se puede buscar la respuesta a la pregunta sobre las funciones de las fronteras en el mundo contemporáneo. $\mathrm{Y}$ sabemos que son un fenómeno mucho más complejo que solo las líneas de separación entre Estados, y que sobrepasan la mera dimensión geográfica. Kalevi Holsti, en sus consideraciones sobre la territorialidad, presenta las funciones básicas de las fronteras. Entre ellas menciona las siguientes: 1) definen los límites territoriales de la jurisdicción legal de los poderes de un Estado, la cual constituye una función crucial para la idea de soberanía; 2) son una especie de barrera para todos tipos de peligros e intrusos indeseados (ejércitos enemigos, terroristas, traficantes de drogas, personas y todos bienes cuya entrada a un Estado debe ser controlada); 3) las fronteras, como ya fue mencionado, tienen la función de identificación de la comunidad cultural, la cual es diferente de otras comunidades ("nosotros" versus "otros") - como indica Holsti, esto coincide a veces con importantes diferencias sociales y culturales, como por ejemplo idioma, religión o historia; 4) al final, él indica la función de control que realizan las fronteras - su funcionamiento ayuda a los Estados y a sus autoridades a controlar el flujo de los bienes, cobrar impuestos y tarifas (Holsti 2004: 95-96). De manera más sintética, las funciones de las fronteras se pueden dividir en las siguientes categorías: función de barrera (protección ante la llegada de intrusos o ideas indeseables), función política (separación de los Estados con diferentes sistemas políticos), función económica (regulaciones del comercio) y función de creación de las zonas fronterizas específicas (Misiak 2003). Una tipología más general de las funciones de las fronteras la proponen Boehmer y Peña. Según ellos, el criterio básico es el nivel de apertura de las fronteras. En consecuencia, se puede decir sobre las funciones que facilitan el cruce
Fronteras como categoría social. La perspectiva teórica de las fronteras en el siglo $X X I$

Karol Derwich 
Dossier América Latina: fronteras y zonas fronterizas y las funciones panópticas en situación cuando el control del flujo de personas o bienes debe ser más restringido (Boehmer, Peña 2012: 274). Sin duda, durante la gran intensificación del proceso de globalización, los Estadosnación pierden parte de su control estricto de las fronteras. No obstante, los gobiernos entienden que la existencia de fronteras, aunque tengan una forma diferente, es fundamental. Hoy día, como antes, las fronteras representan un acto de poder, sea real o simbólico, que construye un fundamento del Estado en las esferas donde sus acciones pueden ser cuestionadas. Esto nos permite percibir las fronteras también como un fenómeno construido como resultado de los procesos sociales. En efecto, regresamos al "proceso de fronterización”, en el sentido de la recreación permanente de las fronteras (Wonders 2007: 33-34). Los académicos que perciben las fronteras como instituciones enfocan su atención sobre su función coordinadora, la cual permite a los actores diferenciar dónde "nosotros" y "otros" comienzan. De esta manera reducen la incertidumbre en varios niveles (Carter, Goemans 2014: 286-287). Las funciones de las fronteras, sin embargo, no tienen solamente el carácter de barrera para separar y proteger. La característica inseparable de las fronteras es que también se las cruza. El movimiento a través de las fronteras puede ser legal o ilegal. Esto fuerza a los gobiernos a realizar actividades que permiten manejar el movimiento fronterizo, o sea, formular políticas y actividades que permitan a los gobiernos usar las fronteras más como fuente de beneficios que como peligros para su población y sus intereses (Newman 2003: 14).

\section{¿Una teoría de las fronteras?}

No se necesita un análisis muy profesional para concluir que las fronteras particulares son muy diferentes. No se puede comparar la frontera entre Israel y la Autoridad Palestina con la frontera entre los países de Benelux. Igualmente, ¿cómo comparar la frontera de los Estados Unidos con México y con Canadá? ¿Cuáles son las similitudes entre la frontera de la India con Pakistán y la que hay entre Brasil y Colombia en la Amazonía? Las fronteras son diferentes, pero lo más importante es que las condiciones de su funcionamiento son diferentes. También vale la pena recordar que las consecuencias del proceso de establecimiento de las fronteras tienen impacto sobre su funcionamiento (Carter, Goemans 2014: 285-302). Entonces, es justificada la pregunta por la teoría universal de las fronteras y las posibilidades de unificación de los estudios sobre ellas. Como lo muestra Eric Tagliacozzo, en la historia de los estudios sobre el fenómeno de las fronteras se pueden encontrar varias aproximaciones. Las más básicas y obvias son las aproximaciones comparativas dentro de la corriente normativa. Son muy importantes y tienen gran valor porque permiten identificar las diferencias y similitudes entre varias fronteras. Esto, por ejemplo, permitió crear una clasificación de las fronteras según 
la cual se puede enumerar: 1) fronteras silenciosas - un Estado y una población local se relacionan de manera pacífica y ambas partes tienen beneficios del funcionamiento de la frontera, 2) fronteras ingobernables - ni un Estado ni élites locales tienen impacto sobre la población local que influye en el funcionamiento de la frontera, la cual puede ser descrita como ingobernable; 3) fronteras rebeldes -élites locales junto con la población local cuestionan decisiones de las autoridades estatales, lo que puede conducir a una confrontación y al nacimiento del separatismo (Tagliacozzo 2015: 4). Como una aproximación "tradicional”, se puede tratar la geográfica. Según los que la usan, las fronteras tienen gran importancia para las relaciones de poder y una compleja economía política. Algunos geógrafos concentran su atención sobre las interacciones entre las fronteras, por ejemplo en la perspectiva espacial, pero también en otras, como en la canalización de flujos de personas o bienes (Tagliacozzo 2015: 4). También existe el concepto de las fronteras desde la perspectiva de Estado como su creador. Esta aproximación se puede encontrar entre algunos representantes de las ciencias políticas. Sin embargo, existen también otras aproximaciones, las cuales pueden ser nombradas "modernas". Entre ellas se enumera la aproximación antropológica, la cual se concentra de manera muy fuerte en las interacciones de las zonas fronterizas con las poblaciones locales. Algunos antropólogos se concentran principalmente en las fronteras sociales, las cuales rigen las relaciones sociales y significan fraccionamiento de las comunidades. Otros se enfocan en las fronteras culturales, que separan varios mundos de significados. Otros más enfocan su atención en las fronteras cuya característica básica es la delimitación del espacio geopolítico. Estos tres elementos, por supuesto, no se excluyen (Donnan, Wilson 2001: 39). Ya en los años noventa del siglo XX, Robert Álvarez indicó que se deben analizar varias fronteras conceptuales, las cuales pueden ser llamadas zonas fronterizas de las prácticas sociales (Álvarez 1995: 448). Su voz es muy importante en la postulación de analizar las fronteras en un contexto social más amplio. Los antropólogos perciben fronteras menos en el aspecto teórico y más como sujeto de análisis a nivel local, empírico. En consecuencia, se identifican tres factores muy importantes en la aproximación antropológica: 1) concentración sobre la dimensión local, 2) enfoque sobre el poder realizado a nivel local y 3) concentración sobre la población local (Tagliacozzo 2015: 6). Aceptando la percepción de fronteras como instituciones que deben ser manejadas, esta aproximación tiene gran importancia. Parece ser imposible el proceso de manejo de fronteras y formulación de las propias políticas sin tomar en cuenta las dinámicas locales.

Stefania Donzelli considera tres aproximaciones que -según ella- tienen el impacto más importante. La primera, la aproximación postmoderna, está concentrada en la función de las fronteras de separar los "otros" de "nosotros". Los temas de exclusión e inclusión dominan en los estudios realizados según esta aproximación. La segunda es la aproximación de gobernabilidad (governmentality approach). Según los que la usan, las fronteras son parecidas a un
Fronteras como categoría social. La perspectiva teórica de las fronteras en el siglo $X X I$

Karol Derwich 
Dossier América Latina: fronteras y zonas fronterizas producto de procesos gubernamentales que pueden ser estudiados enfocándose en las actividades prácticas y también en la idea de soberanía. Al final, la autora menciona la aproximación de la economía política. Aquí las fronteras no son percibidas solo en el sentido de una simple división, sino como un sitio de tensiones entre los procesos coexistentes, los procesos tanto de separación como de conexión. Se intenta sobrepasar la dicotomía de exclusión/ inclusión y se enfatiza la multiplicación de las posiciones de sujetos que están en movimiento (Donzelli 2013: 7-14).

Ente otras aproximaciones modernas, Tagliacozzo enumera la ecológica, etnográfica, legal, lingüística, o la teoría de la neodependencia (Tagliacozzo 2015: 7). Sin duda, lo que puede ser una de las características más importantes de los estudios sobre las fronteras es la multitud de aproximaciones y la gran variedad de conceptos metodológicos que son consecuencia del interés en las fronteras entre los representantes de varias disciplinas. Al dejar de percibir las fronteras solo como unas líneas divisoras y ante el creciente interés en su "ámbito", hay una gran necesidad de llevar a cabo análisis conducentes al entendimiento más amplio de las fronteras. Una consecuencia de este proceso es el desarrollo de los estudios sobre fronteras que usan varias aproximaciones y atraen gran atención a las zonas fronterizas. Se enfoca la atención en los actores no centrales y no estatales, en las comunidades plurinacionales que disminuyen la impermeabilidad de las fronteras e incrementan su carácter poroso (Brunet-Jaily 2005: 639).

La variedad de las aproximaciones de los estudios sobre las fronteras conlleva la pregunta sobre la selección de la teoría unificada de las fronteras más importante. No obstante, la respuesta es bastante problemática. ¿Cómo crear una teoría del fenómeno tan diferente, analizado desde tantos puntos de vista? En este sentido parece ser correcta la duda que presenta Anssi Paasi al respecto de la posibilidad de formular una teoría universal de las fronteras. Según él, hay que tomar en cuenta una gran contextualización del fenómeno de las fronteras. No se puede analizar y estudiar las fronteras sin poner atención al contexto sociocultural de su funcionamiento. Hay muchas características y procesos de naturaleza diferente - puede ser política, social, cultural, etc. - que deben ser tomados en cuenta y analizados en contextos más amplios. Junto con este aumento del entendimiento de las fronteras aparecen nuevas categorías que influyen en el proceso de análisis de las mismas. Se puede mencionar en este lugar, entre otros, las funciones y formas del poder de Estado, las tendencias geopolíticas y geoeconómicas, las formas de control social y político, la seguridad o el medio ambiente (Paasi 2009: 223-224). En consecuencia, se debe decir, de manera muy expresa, que los estudios fronterizos contemporáneos están evolucionando desde los estudios sobre la dimensión territorial o geográfica de fronteras hacia su percepción como un fenómeno mucho más complejo. Hoy día es deseado tratar los estudios fronterizos como estudios sobre las prácticas humanas que constituyen y representan las diferencias en espacio (van Houtum 2005: 672). 
La percepción de frontera como una acción, o sea, el "proceso fronterizo", tiene gran razón y justificación. En consecuencia, en los estudios fronterizos contemporáneos es inseparable no solo el análisis del carácter de la geografía política, sino también se requieren investigaciones en el área de sociología, economía y cultura. Esto parece ser natural como consecuencia de la adopción de la noción de frontera como un factor que separa las comunidades diferentes. Aunque se puede también encontrar una función creativa de las fronteras, o sea, las fronteras pueden ser simultáneamente las instituciones que separan, pero también las que crean nuevos espacios, con comunidades y características distintas del resto de la comunidad en ambos lados de la frontera. Esta función es enfatizada por Etienne Balibar, quien escribe sobre la frontera como un factor que crea gente, idiomas, etc. En consecuencia de la función "creativa" de las fronteras se construyen las zonas transnacionales, las cuales incluyen territorios, población y prácticas de ambos lados de la frontera (Balibar 2010: 316). La creatividad de las fronteras, entendida como la explica Balibar, es la otra voz que justifica y permite percibirlas como constructos no solo territoriales sino también sociales. Todo eso nos conduce al punto en el cual la diferencia entre la dimensión ontológica (búsqueda de la respuesta a la pregunta ¿qué son las fronteras?) y epistemológica (la respuesta a la pregunta ¿qué sabemos sobre las fronteras?) se desvanece. La convicción de que las fronteras son "productos" sociales, o sea, se construyen en efecto de las prácticas sociales, permitió a los estudios fronterizos dejar de tratar las fronteras como un mero atributo de Estados (van Houtum 2005: 674).

La conciencia del análisis contextual de las fronteras percibidas como constructos sociales es muy importante en el mundo contemporáneo. Junto con la intensificación significante del proceso de globalización, entramos al mundo en el cual las fronteras nos afectan en muchas situaciones. Como ya hemos indicado, la globalización no conduce al mundo sin fronteras. Más bien, las fronteras están más cerca de los individuos que pueden tener más facilidad para cruzarlas. Ya no son unas líneas en las periferias del Estado que principalmente sirven para separar una comunidad de otra. Esta cercanía de las fronteras resulta en un proceso de social spatialization. Esta noción enfoca el papel de la población en el proceso de formación espacial (Paasi 2009: 226). En efecto, se puede concluir que la interacción entre la frontera y la población sucede en ambas direcciones. Los análisis de las zonas fronterizas particulares muestran que la frontera tiene gran importancia para la población que vive en estas zonas y tiene contacto diario con la frontera. Lo mismo se puede decir sobre los individuos que cruzan fronteras, por ejemplo, migrantes o refugiados. Ellos entran a un espacio ya definido mientras que son extranjeros en este espacio. En consecuencia, tiene lugar la reunión de "nosotros" con los "otros". Para mejor estudio de las fronteras, entendidas de dicha manera, Paasi recomienda usar -entre otros- la aproximación metodológica conocida como
Fronteras como categoría social. La perspectiva teórica de las fronteras en el siglo $X X I$

Karol Derwich 
Dossier América Latina: fronteras y zonas fronterizas representación social (Paasi 2009: 228). Según este autor, esto también es una evidencia de que es imposible efectuar análisis adecuados y completos de las fronteras formulando una teoría universal. La multidimensionalidad de las fronteras requiere usar varias teorías y metodologías diferenciadas.

\section{La apertura y los muros}

La conciencia de las fronteras como constructos sociales, en las cuales las comunidades juegan un papel importante, tiene gran valor en un mundo globalizado, cuando el proceso de apertura es muy intenso. En muchos casos esto se refiere no solo a los bienes y servicios sino también a las personas. Boehmer y Peña presentaron los factores decisivos para la apertura de fronteras. Entre ellos se pueden diferenciar los que se refieren a la seguridad, la economía o el sistema político. Las siguientes conclusiones resultan de su análisis. Primero, el nivel de riqueza entre los Estados vecinos tiene influencia sobre sus fronteras y la escala de su apertura. El Estado está más ansioso por abrir su frontera, si su vecino tiene un alto nivel de riqueza. Cuanto más pobre el vecino, más restrictivo es el Estado respecto a la apertura de su frontera. En general, la probabilidad de abrir la frontera para los ciudadanos del país vecino es proporcional al nivel de su riqueza. Simultáneamente, cuando la desproporción es más grande, el país más pobre estará más abierto para los ciudadanos del vecino rico. Una observación muy interesante de los autores dice que la existencia de las ciudades fronterizas apoya la apertura de las fronteras y no importa la desproporción en desarrollo. Un ejemplo de esta situación puede ser la zona fronteriza entre los Estado Unidos y México y el funcionamiento de las ciudades fronterizas como San Diego-Tijuana, El Paso-Ciudad Juárez, Nogales en los Estado Unidos y Nogales en México. La apertura de las fronteras también depende de la historia de las relaciones entre los dos países. Si un Estado sufrió una agresión por parte de su vecino, no va a permitir el cruce de ciudadanos de ese país por su frontera. Los factores que apoyan el proceso de apertura de las fronteras son también el idioma común y el funcionamiento del régimen democrático en ambos países (Boehmer, Peña 2012: 279-282). La ejemplificación más clara de la influencia de estos factores al proceso de liberalizar las fronteras es la cooperación entre los países de la Unión Europea formando el Espacio de Schengen. En América Latina también es posible encontrar casos que confirman los resultados de la investigación hecha por Boehmer y Peña. Ya se mencionaron las ciudades fronterizas entre los Estado Unidos y México. Las reglas del movimiento de personas entre los países que forman el Mercosur confirman que el comercio más avanzado entre los países y el apoyo a los valores democráticos compartido en general entre los miembros de dicha iniciativa regional está de acuerdo con los efectos de la investigación hecha por los autores.

Como ya se ha indicado, la intensificación de la globalización no significa la muerte de los Estados-nación y tampoco significa la desaparición de las 
fronteras. La facilidad de cruzarlas, tanto por los bienes como por las personas, solamente es resultado del cambio de su naturaleza, carácter y percepción. Es la globalización y los cambios en el funcionamiento de las fronteras lo que hace que cambie su naturaleza desde una línea de separación hacia una zona de funcionamiento de dos comunidades. Y son las comunidades fronterizas las que impactan en el carácter de la frontera. Aunque la frontera sea manejada principalmente por las autoridades centrales de los Estados que formulan políticas respecto a las relaciones con el país vecino y la política exterior en general, el impacto de las comunidades locales y las autoridades locales juega un papel significativo. Esto es otra evidencia de la necesidad de entender las fronteras como fenómenos sociales. Una de las consecuencias de la globalización observada en las zonas fronterizas es la creación de lo que se puede describir como zonas transfronterizas o transnacionales. Como consecuencia de la creciente apertura de la frontera, las comunidades a ambos lados entran en interacción de varias maneras: económicas, sociales, culturales. En efecto, una comunidad puede adaptarse a las características de otra y viceversa. Como consecuencia de estas interacciones puede desarrollarse -en ambos lados de la frontera- una comunidad transnacional mientras que la zona fronteriza cambia a una de carácter transfronterizo. Si un individuo vive en la zona fronteriza, tiene contacto casi constante con los elementos de los "otros" cuando cruza la frontera o ellos llegan a su lado. En el contexto de la globalización estas interacciones pueden tener una intensidad muy fuerte. En estas situaciones está claro que las prácticas similares aparecen en los dos lados de la frontera (Vila 2003: 611). Podemos observarlo en la frontera -entendida en la manera más amplia como zona fronteriza- entre México y los Estados Unidos. Especialmente en lugares donde existen ciudades fronterizas. Durante sus investigaciones el autor pudo observar este fenómeno en Ciudad Juárez-El Paso y Tijuana-San Diego. Los ejemplos más obvios del funcionamiento de una comunidad transnacional en dichas áreas son la popularidad de la comida mexicana al norte del río Bravo y la creación de lo que se denomina como tex-mex. Pero la hibridación de la comunidad de la zona fronteriza es mucho más fuerte. También los vínculos económicos refuerzan la conciencia de cierta comunidad. Todo esto no excluye la percepción de los "otros". Muchos norteamericanos todavía presentan posturas antimexicanas, y, en ciertas situaciones, racistas. El sentimiento antiinmigración entre los ciudadanos de los estados fronterizos de los Estados Unidos está presente. También los mexicanos que viven en la zona fronteriza tienen una identidad nacional fuerte. Entendiendo la existencia de los vínculos mutuos y las interacciones que tienen lugar, todavía perciben a los vecinos del otro lado de la frontera como "gringos".

Entonces, el nivel más grande de interacciones entre las comunidades fronterizas, que es una de las consecuencias de la globalización, no significa automáticamente un mundo sin fronteras. Las observaciones del mundo contemporáneo pueden conducirnos a la tesis de que, por causa del aumento de los flujos transfronterizos, algunos líderes y representantes de comunidades
Fronteras como categoría social. La perspectiva teórica de las fronteras en el siglo XXI

Karol Derwich 
Dossier América Latina: fronteras y zonas fronterizas formulan postulados de cerrar las fronteras y tratarlas de nuevo, y principalmente, como una barrera. Este regreso a la percepción de las fronteras como muros -a veces literalmente- está bien ilustrado en la crisis migratoria en Europa. Después del inicio de la llegada de la gran ola de emigrantes musulmanes del norte de África y Medio Oriente, algunos gobiernos europeos buscaron, muy nerviosos, una solución para bloquear la entrada de dichos emigrantes al territorio de sus Estados. Por ejemplo, el gobierno de Hungría construyó un muro en forma de valla en su frontera con Serbia. En la frontera sur de los Estados Unidos, la idea del muro no es nada nuevo, aunque durante la campaña presidencial de 2016, el candidato del Partido Republicano, Donald Trump, hizo de la idea del muro y la separación de los Estados Unidos de México uno de los postulados fundamentales de su campaña, y sigue manteniendo este postulado ya como presidente. La frontera entre los dos países es, otra vez, un buen ejemplo de la presencia de diferentes factores que se deben tomar en consideración cuando se busca la respuesta a la pregunta sobre las fronteras en el mundo globalizado. Un análisis de los motivos por los cuales las autoridades estadounidenses quieren construir una barrera física en su frontera sur muestra también las funciones de las fronteras y las esperadas posibilidades de usar las fronteras para lograr varios objetivos. En el caso de esta frontera en particular, el factor principal es la seguridad nacional. Para las autoridades estadounidenses, México es una fuente de varios peligros. Primero, es una fuente de drogas que entran al territorio de los Estados Unidos. Segundo, es una fuente -al menos según algunos políticos en los Estados Unidos- de violencia, la cual es consecuencia del funcionamiento de grupos del crimen organizado en México y la lucha entre ellos y las fuerzas de seguridad mexicanas. Tercero, después de los ataques terroristas del 11 de septiembre de 2001, el gobierno estadounidense decidió sellar la frontera para prevenir la entrada de los miembros de grupos terroristas (CorreaCabrera, Garrett, Keck 2014: 35-53). Donald Trump añadió a estos factores "tradicionales" unos de la naturaleza social y económica. Según él, junto con una política tan restrictiva respecto a los inmigrantes, el muro debe servir como una barrera para detener el flujo de migrantes latinoamericanos a los Estados Unidos. Esto coincide con el factor económico. Los inmigrantes de América Latina y el Caribe ocupan lugares de trabajo de los ciudadanos estadounidenses. Entonces, en este caso es evidente el regreso a la percepción de la frontera como una barrera que no solo separa "nosotros" de los "otros", sino también nos defiende en una situación de multiplicidad de peligros. La pregunta es si en el mundo globalizado, en el que una de sus características es la facilidad de transporte, es posible usar las fronteras como barreras para separar. Desde el punto de vista puramente práctico, parece que sí. En siglo XXI no faltan muros físicos en las fronteras. El presidente Trump habla sobre la construcción de un muro en la frontera sur de los Estados Unidos, pero de hecho lo que quiere hacer es concluir la construcción que ya está presente en varios fragmentos de la frontera. Otro ejemplo de muros -el que se encuentra 
entre Israel y Palestina- confirma que la construcción de barrera física no es ningún problema. Su construcción se inició durante la Segunda Intifada, en 2003, y según datos de la ONU, cuando se haya completado tendrá una longitud de 700 kilómetros. Según las autoridades israelíes, desde que se levantara el muro, ningún asesino suicida palestino ha sido capaz de cruzar a su territorio. Lo que para unos es una barrera de seguridad para otros significa un elemento marginador. Entonces, si la construcción física no es un problema, la siguiente pregunta es sobre los costos. En el caso del muro norteamericano, la Iniciativa de la Frontera Segura (The Secure Border Initiative -SBI) incluye la construcción del muro de 670 millas de longitud, desde Brownsville, Texas, hasta San Diego, California, con un costo de 3 mil millones de dólares y una valla virtual en Arizona, la cual costaría más de 1,5 mil millones de dólares (Correa-Cabrera, Garrett, Keck 2014: 42). Son costos demasiado altos. La cuestión financiera respecto a la construcción del muro confirma los problemas de la administración del presidente Trump y su presión al gobierno mexicano sobre su participación para financiar la construcción de dicho muro. Pero lo que no se puede perder de vista son también los costos no financieros. La creación de una frontera tan restrictiva y difícil para cruzar puede generar nuevos problemas. Ya se puede observar el empeoramiento de las relaciones bilaterales entre México y los Estados Unidos. La crisis en las relaciones, por supuesto, no es solamente efecto de la idea del muro, sino que el simple tema de su construcción genera muchas emociones en la sociedad mexicana, y su efecto inicial es el fortalecimiento de la percepción negativa de los "otros" que viven al norte del río Bravo. Es muy probable que el muro no vaya a disminuir ni el tráfico de drogas ni la inmigración ilegal. Solamente puede crear prácticas más sofisticadas del tráfico de bienes y personas ilegales. Esto, en consecuencia, puede conducir a un incremento de la violencia en la zona fronteriza, de tal forma que las confrontaciones entre las fuerzas fronterizas de los Estados Unidos y quienes cruzan la frontera ilegalmente ocurran con más frecuencia (Carter, Poast 2017: 264).

\section{Conclusiones}

La vuelta de unos políticos y líderes a la percepción de las fronteras principalmente como lo que separa y construye una barrera para proteger el país frente a varios peligros es el tipo de pensamiento reducido a solo una perspectiva de entenderlas. Lo que falta es un contexto más amplio. Las interacciones entre ambos lados de la frontera pueden ser, en el mundo contemporáneo, tan fuertes y cercanas que no vayan a desaparecer en el proceso de manejar las fronteras. La aproximación a este proceso tiene que tomar estas interacciones en cuenta. En consecuencia, si en la práctica no es posible reducir el fenómeno de la frontera a una mera línea de separación, la teoría de las fronteras debe tratarlas como fenómenos sociales.
Fronteras como categoría social. La perspectiva teórica de las fronteras en el siglo XXI

Karol Derwich 
Dossier América Latina: fronteras y zonas fronterizas
El proceso de la globalización no está en contra del fenómeno de la existencia de fronteras. Como he mostrado, es posible una conclusión contraria -la globalización puede fortalecer este fenómeno, pero no por hacer las fronteras más restrictivas y convertirlas en barreras difíciles de cruzar, pero por hacerlas un fenómeno más vivo, un lugar donde ocurren varias interacciones de natura muy diferente. Hoy día las fronteras no son solamente muros y campos minados, como en el caso de la frontera entre Israel y Palestina, o las Coreas. Se debe entender las fronteras en su aspecto más amplio. Es importante tenerlo en cuenta, porque esto permite hacer estudios sobre las fronteras más completos y comprender mejor los procesos que ocurren en las zonas fronterizas.

\section{Referencias bibliográficas}

Alvarez R. R. (1995) The Mexican-US Border: The Making of an Anthropology of Borderlands, "Annual Review of Anthropology", vol. 24, pp. 447-470.

Anderson J., O’Dowd L. (1999) Borders, Border Regions and Territoriality: Contradictory Meanings, Changing Significance, "Regional Studies", vol. 33, pp. 593-604.

Balibar E. (2010) At the borders of citizenship. A democracy in translation, "European Journal of Social Theory", vol. 13, no 3, pp. 315-322.

Boehmer Ch. R., Peña S. (2012) The Determinants of Open and Closed Borders, "Journal of Borderlands Studies", vol. 27, no 3, pp. 273-285.

Brunet-Jailly E. (2005) Theorizing Borders: An Interdisciplinary Perspective, "Geopolitics", vol. 10, pp. 633-649.

Carter D. B., Goemans H. E. (2014) The temporal dynamics of new international borders, "Conflict Management and Peace Science", vol. 31, no 3, pp. 285-302.

Carter D. B., Poast P., (2017) Why Do States Build Walls? Political Economy, Security, Border Stability, "Journal of Conflict Resolution", vol. 6, no 2, pp. 239-270.

Correa-Cabrera G., Garrett T., Keck M. (2014) Administrative Surveillance and Fear: Implications for U.S. - Mexico Border Relations and Governance, "European Review of Latin American and Caribbean Studies", no 96, pp. 35-53.

Hastings D., Wilson T. M. (2001) Granice tożsamości, narodu, państwa, Wydawnictwo UJ, Kraków.

Donzelli S. (2013) Border Studies. Theoretical Approaches, Themes of Inquiry, and Suggestions for Future Works, International Institute of Social Studies of Erasmus University, Working Paper, no 571, "Migration Literature Review", no 3, pp. 1-52.

Held D., McGrew A., Goldblatt D., Perraton J. (1999) Globalization, "Global Governance", vol. 5 , no 4 , vol. 5 , no 4 .

Holsti K. J. (2004) Taming the Sovereigns. Institutional Change in International Politics, Cambridge University Press, Cambridge.

Kissinger H. (2002) Does America Need a Foreign Policy? Toward a Diplomacy for the $21^{\text {st }}$ Century, Touchstone Books, New York. 
Li Ben G., Penglong Z. (2017) International Geopolitics, Boston College, disponible en: https://www2.bc.edu/ben-li/GEO.pdf

Misiak W. (2003) Funkcje granic, en: Transgraniczność w perspektywie socjologicznej. Teorie, studia, interpretacje, M. Zielińska (ed.), Lubuskie Towarzystwo Naukowe, Zielona Góra.

Naím M. (2009) Globalization, "Foreign Policy”, no 171.

Newman D. (2003) On Borders and Power: A Theoretical Framework, "Journal of Borderlands Studies", vol. 18, no 1, pp. 13-26.

Newman D. (2006) The Lines That Continue to Separate Us: Borders in Our 'Borderless' World, "Progress in Human Geography", no 30, pp. 186-207.

Paasi A. (2009) Bounded Spaces in a 'Borderless World': Border Studies, Power and the Anatomy of Territory, "Journal of Power", vol. 2, issue 2, pp. 213-234.

Rosenau J. (1997) Along the Domestic-Foreign Frontier: Exploring Governance in a Turbulent World, Cambridge University Press, Cambridge.

Stiglitz J. E. (2004) Globalizacja, Wydawnictwo Naukowe PWN, Warszawa.

Tagliacozzo E. (2015) Jagged in the Landscape: Conceptualizing Borders and Boundaries in the History of Human Societies, "Journal of Borderlands Studies", vol. 31, no 1, pp. 1-20.

van Houtum H. (2003) The Geopolitics of Borders and Boundaries, "Geopolitics", vol. 10, 2005, pp. 672-679.

Vila P. (2003) Processes of Identification on the US-Mexico Border, "The Social Science Journal", vol. 40, pp. 607-625.

Walther O. J., Retaillé D. (2014) Rethinking Borders in a Mobile World: An Alternative Model, Department of Order Region Studies Working Paper Series, Working Paper no 3.

Wonders N. A. (2007) Order Reconstruction Projects and Transnational Crime, "Social Justice", vol. 36, no 4.

Zachara M. (2012) Global governance. Ład międzynarodowy po zakończeniu stulecia Ameryki, Wydawnictwo UJ, Kraków.
Fronteras como categoría social. La perspectiva teórica de las fronteras en el siglo $X X I$

Karol Derwich 